\title{
SAR AND OBLIQUE AERIAL OPTICAL IMAGE FUSION FOR URBAN AREA IMAGE SEGMENTATION
}

\author{
Julian Fagir, Adrian Schubert, Max Frioud, Daniel Henke
}

Remote Sensing Laboratories, University of Zürich, CH-8057 Zürich, Switzerland - firstname.lastname@ geo.uzh.ch

KEY WORDS: Remote Sensing, Urban Area, Image Fusion, Image Segmentation, SAR, RGB Image

\begin{abstract}
:
The fusion of synthetic aperture radar (SAR) and optical data is a dynamic research area, but image segmentation is rarely treated. While a few studies use low-resolution nadir-view optical images, we approached the segmentation of SAR and optical images acquired from the same airborne platform - leading to an oblique view with high resolution and thus increased complexity. To overcome the geometric differences, we generated a digital surface model (DSM) from adjacent optical images and used it to project both the DSM and SAR data into the optical camera frame, followed by segmentation with each channel. The fused segmentation algorithm was found to out-perform the single-channel version.
\end{abstract}

\section{INTRODUCTION}

The amount of readily available SAR and optical data has been rapidly increasing in recent times, making the creation of data products using SAR / optical image fusion an interesting research topic that can support extensions, or improve existing methods. However, image segmentation based on the joint use of SAR and optical data is a rarely treated topic. A closely related area, classification, has had some success, although the literature mostly concentrates on nadir-view RGB images, e.g. (Voisin et al., 2014) or (Gaetano et al., 2008).

When both the SAR and the optical sensor are mounted on the same airborne platform, facing the same direction, the optical images will be in oblique view at a very high resolution. This increases the geometric differences between SAR and optical even more than nadir-view, but also means that both sensors see the same surfaces. This situation is uncommon and it complicates fusion-related work, but also enables new aspects, as e.g., façade detection (Schack et al., 2015). The geometric differences can also be used for 3D reconstruction via stereogrammetry (Wegner and Soergel, 2008, Sportouche et al., 2011), as multiple views of a scene may improve classification (Boulch et al., 2017). But without a digital surface model (DSM), the different geometries prevent a direct pixel-based fusion of the two data sources.

In this work, we perform a segmentation of data from the MIRANDA35 SAR and the IGI DigiCAM RGB (red/green/blue) camera mounted on the same platform (Frioud et al., 2014). The approach is related to the optical-guided despeckling from (Verdoliva et al., 2015), where an optical image was used as a pilot for guiding speckle removal of a SAR image. A DSM for geometry compensation is generated photogrammetrically from adjacent overlapping RGB images. The remaining task involves the fusion of segmentation results from very high-resolution SAR and RGB, along with a low-resolution DSM. Segmentation of $\mathrm{RGB}$ is performed with the $\mathrm{gPb}$-owt-ucm pipeline from (Arbelaez et al., 2011). The SAR image is focussed directly on the generated DSM to avoid interpolation and reprojection from/to slant or ground range. This point cloud is projected into the RGB frame to reweight the RGB ucm by comparing the distributions of neighboring regions. The DSM refines the RGB ucm in the same
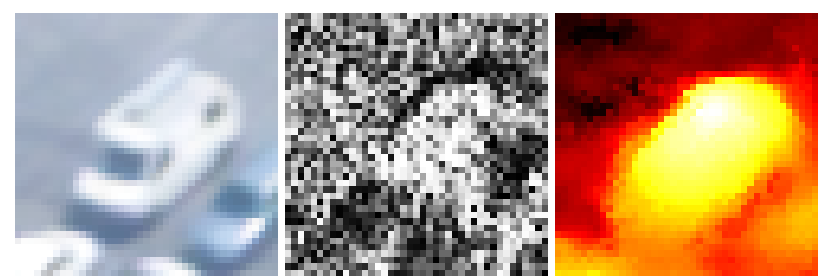

Figure 1. A crop of the van in the top right of figure 2. From left to right: RGB, SAR, DSM. The DSM is depicted in altitude. SAR and DSM are projected into RGB view.

manner (also projected into RGB view), but with the distance of the average normal vectors used as a criterion. The two ucms generated from SAR and the DSM are then intersected for the final output of the segmentation. In contrast to the ucm output from the regular pipeline as applied to RGB, the output is also thresholded by the weights of the edges in the SAR and DSM projections.

We first describe the test site in section 2, followed by the segmentation steps. In section 3 , the results of our experiment on the test site are shown and subsequently discussed in section 4 . Section 5 concludes our work.

\section{METHOD}

The test site under consideration was a parking lot scene in Emmen (LU), Switzerland, depicted in figure 2. The scene analyzed was $300 \mathrm{Px} \times 300 \mathrm{Px}$ (RGB frame) that consisted mainly of asphalt in the background and 97 cars (partially or fully visible) parked on it, colored mainly black, white and silver. Figure 1 is a close-up of the van in the top right of the image in each imaging modality. The sensors flew at an altitude of $1150 \mathrm{~m}$ above ground, the camera and the SAR had a depression angle of about $35^{\circ}$. The resolution of $40 \mathrm{MPx}$ for the IGI DigiCAM resulted in an on-ground resolution of about $20 \mathrm{~cm}$ for RGB (the local resolution depends on the position in the image). The MIRANDA35 SAR was operating at a system bandwidth of $600 \mathrm{MHz}$ (in slant range resolution corresponding to $12 \mathrm{~cm} \times 10 \mathrm{~cm}$ for azimuth, range). 
The proposed segmentation fusion algorithm consists of five steps:

1. Generation and referencing of the DSM.

2. SAR image focussing

3. Projection of SAR and DSM into the optical camera frame.

4. Contour detection and segmentation for RGB.

5. Thresholding of SAR and DSM distances based on the prior RGB segmentation.

Step 1 is a preparatory step before the actual algorithm is applied. The DSM is generated photogrammetrically with the software Agisoft PhotoScan Pro from subsequent images of the RGB camera. A rough referencing is performed with GPS-measured ground control points (GCPs). The airplane orientation is measured with a GPS and an IMU (Inertial Measurement Unit), but the geometric calibration is not precise enough to work with these measurements alone. Thus, fine referencing is also done by matching the DSM with the SAR and the RGB images, keeping the height of the selected GCPs, but rotating and translating the point cloud such that a single image suffices for reference. For the DSM, the normals for each point are generated by performing a principal component analysis (PCA) on all points in a fixed environment around it and then taking the third component as the normal vector.

Focussing of the SAR image is done in step 2. With the time domain processor, the SAR image can be focussed directly onto a given digital elevation model (Frioud et al., 2014). Since the DSM is available as a point cloud, the image is directly focussed on that with a single look.

In the following step 3, the different reference systems must be transformed into a common one to be able to fuse the segmentation. The DSM and SAR are available in WGS84, the camera orientation for RGB is given by the onboard GPS (in WGS84) and IMU (in roll/pitch/yaw). The DSM and the SAR point clouds are back-projected into the image reference system. The phase and intensity values for the SAR and for the DSM the normals (component-wise) associated with each point are interpolated linearly to the RGB image frame.

For step 4, RGB is segmented with the $\mathrm{Pb}$ contour detector, which proved to be one of the best-performing algorithms in its framework (Arbelaez et al., 2011). Pb analyzes the histogram of an environment around each pixel. If the histogram is split in two along a line through the middle pixel, the saliency value for a pixel is then the $\chi^{2}$-distance between the histograms of the left and the right part. By changing the direction along which left and right are defined, orientation is introduced. The following oriented watershed transform (owt) is a watershed transform that weights each detected boundary by the underlying contour, determined by the orientation. The ultrametric contour map (ucm) is a hierarchical segmentation from a weighted segmentation, providing a tuneable parameter for changing the degree of fineness of the segments. For every pair of two neighboring regions, the boundary is assigned a weight describing the probability for its existence, i.e., the probability that these two regions are really separate. By thresholding the probability, the level of detail of the segmentation can be selected.

The motivation behind the fusion in step 5 is to take into consideration the different scales in which the datasets work. Even though they formally have the same resolution, the information content is different. Given a camera with comparable spatial resolution, RGB will show fine details, while speckle noise and certain kinds

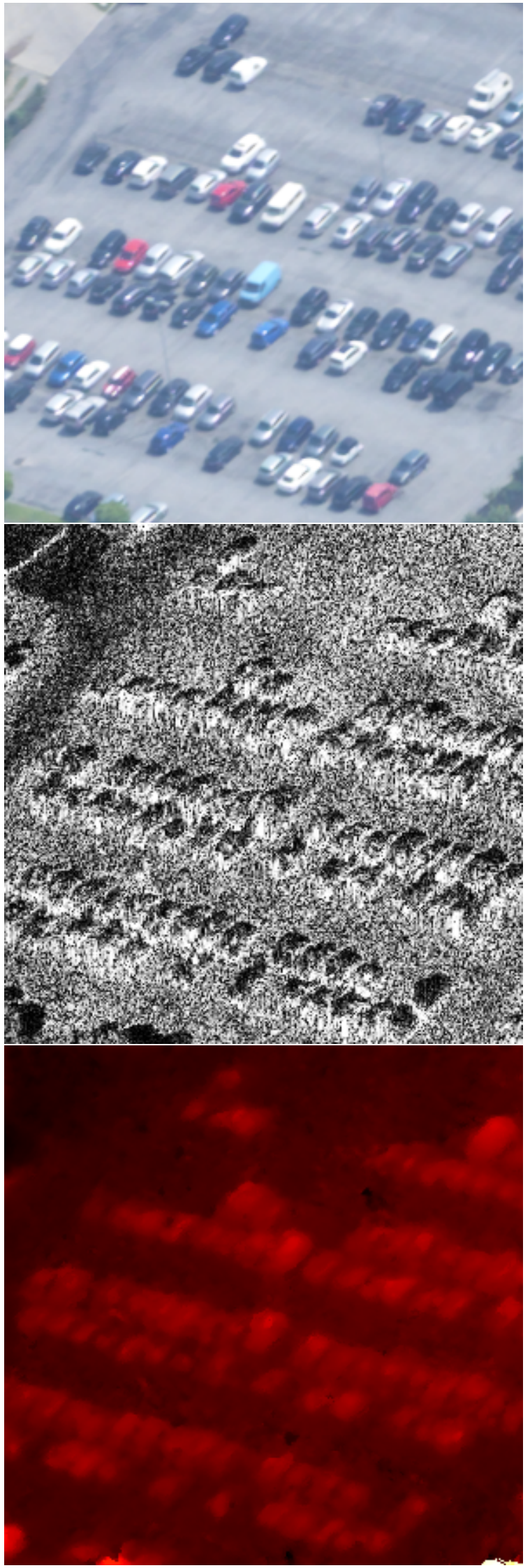

Figure 2. Top: RGB image. Middle: SAR image, projected in RGB view. Bottom: Elevation map of the used DSM ranging from $429 \mathrm{~m}$ (black) over red and yellow to $432 \mathrm{~m}$ (white), projected into optical camera view. 
of artefacts make the detail in SAR images at the same resolution more difficult to interpret in practice. The DSM has a very high precision but a low resolution, and the required filtering makes it smoother than desired.

Let $u c m_{\mathrm{RGB}}$ be the ucm from the RGB image; then two new contour maps $u c m_{\mathrm{SAR}}\left(u \mathrm{~cm}_{\mathrm{RGB}}\right)$ and $u c m_{\mathrm{DSM}}\left(u c m_{\mathrm{RGB}}\right)$ are generated to describe the weight of the edges in the SAR and DSM domains, resp. The weight of an edge in $u \mathrm{~cm}_{\mathrm{SAR}}$ is evaluated by the distributions of the two neighboring regions. For a boundary under consideration separating regions $r$ and $s$, the amplitude distributions are binned with the same bins to get the histograms $h_{r}$ and $h_{s}$ of the corresponding regions. The weight of the boundary is set to the $\chi^{2}$-distance between $h_{r}$ and $h_{s}$ :

$$
d(r, s)=\sum_{b \text { bin }} \frac{\left(h_{r}(b)-h_{s}(b)\right)^{2}}{h_{r}(b)+h_{s}(b)}
$$

For a region $r$ in $u c m_{\mathrm{DSM}}, n_{r}$ is the mean normal vector of all points in $r$. The boundary weight between regions $r$ and $s$ is defined as the great-circle distance between $n_{r}$ and $n_{s}$. For both SAR an DSM, the contour map is generated by iteratively merging the two regions $r_{m}, s_{m}$ with minimal distance, the weight of the boundary is set to $d\left(r_{m}, s_{m}\right)$, until only one region is left.

For the fusion, three thresholds must be chosen: $t_{\mathrm{RGB}}, t_{\mathrm{SAR}}, t_{\mathrm{DSM}}$. The first one is used to threshold $u \mathrm{~cm}_{\mathrm{RGB}}$ before the generation of the other ucms. Let $u \mathrm{~cm}^{t}$ denote all edges in the contour map $u \mathrm{~cm}$ that have a probability of existence higher than $t$. Thus, we generate the two ucms

$$
\begin{aligned}
u c m_{\mathrm{SAR}}^{\prime} & =u c m_{\mathrm{SAR}}\left(u c m_{\mathrm{RGB}}^{t_{\mathrm{RGB}}}\right), \\
u c m_{\mathrm{DSM}}^{\prime} & =u c m_{\mathrm{DSM}}\left(u c m_{\mathrm{RGB}}^{t_{\mathrm{RGB}}}\right) .
\end{aligned}
$$

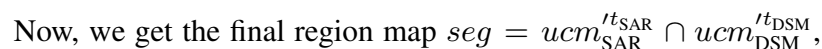
that is, all edges that exist in both $u \mathrm{~cm}_{\mathrm{SAR}}^{\prime t_{\mathrm{SAR}}}$ and $u \mathrm{~cm}_{\mathrm{DSM}}^{\prime t_{\mathrm{DSM}}}$. Edges that do not separate any regions in seg are removed and added to the surrounding region.

The performance of the segmentation fusion is assessed by comparison with ground truth by the segmentation covering measure (Arbelaez et al., 2011). This measure takes the region from the segmentation that has the highest overlap for every ground truth region, and compares the ratio of the overlap to the size of the two original regions. Since the asphalt (black in fig. 3) is easy to segment and makes up roughly $61 \%$ of the image, making the coverage of single cars negligible, it is not evaluated. Ground truth (fig. 3) was determined by a human observer, instructed to mark whole cars. For both segmentations, the thresholds giving optimal results were determined.

\section{RESULTS}

The resulting ucms of the fused and the RGB-only segmentation can be seen in figure 3 . The higher the value of an edge, the more likely it is to exist. Segmentation covering values not including asphalt are $27.0 \%$ for the fused result and $26.5 \%$ for RGB-only, corresponding to a segmentation covering of $64.4 \%$ or $64.3 \%$ including asphalt. Two out of 97 cars (about $2 \%$ ) could be detected significantly better and four out of 97 cars (4\%) could be detected slightly better with the fused segmentation compared to RGB-only. The results are shown in figure 4: For each region of the ground truth (figure 3), the corresponding selected region is transparently overlaid over the RGB image. The failmaps below are the RGB image again with all failed pixels transparently
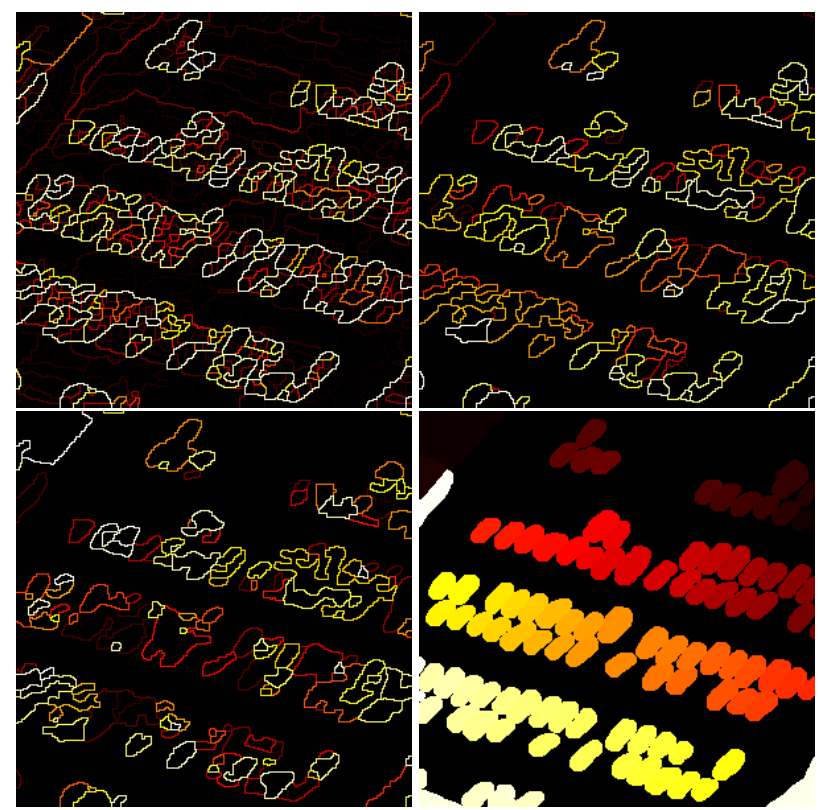

Figure 3. ucms, ranging from black (low probability) over red and yellow to white (high probability). From top to bottom, left to right: RGB-only ucm, DSM-weighted ucm, SAR-weighted $\mathrm{ucm}$, ground truth labels.

overlaid in red. The failmap for the fused results also have the regions that are failed in RGB, but not in the fused segmentations, overlaid in green. It is noteworthy that the ground truth is defined via regions, while the segmentation is based on boundaries, which are not part of a region. Thus, even a perfect segmentation would not have a segmentation covering of $100 \%$, but have the boundaries marked as fails. This is a limitation of the validation process, and not the method itself.

In the results shown, the differences between the segmentations become obvious: RGB barely distinguishes dark cars due to their low contrast with the asphalt. SAR and DSM based segmentation, in contrast, find their outlines clearly. In the fused results, the mixture of the segmentation results does not degrade the ability to distinguish different objects and categories. However, as is visible in figure $3, \mathrm{RGB}$ is still preferred for characterizing the brightly colored cars.

One problem with the fusion method became clear: When the threshold $t_{\mathrm{RGB}}$ is too high, the separating edges are not available anymore. In the top of the fusion image, the large area over the three dark cars illustrates exactly this problem, as can be seen from the ucms in figure 3 . The windshield from the van depicted in figure 1 is also not included in the thresholded ucm used as a basis for $u c m_{\mathrm{SAR}}^{\prime}$ and $u c m_{\mathrm{DSM}}^{\prime}$.

\section{DISCUSSION}

The segmentation based on multiple sensors provides better results than the RGB channel alone. We found that the airborne RGB images provide the highest level of detail, but high-level object contours are indifferently strong as low-level object contours and colors confuse object borders. DSM and SAR products, in contrast, provide useful information about the geometry and surface roughness, which is especially important in urban areas. But they also contain complementary information: While SAR captures even small changes and edges caused by multiple bounces or casting shadows, the photogrammetry-generated 


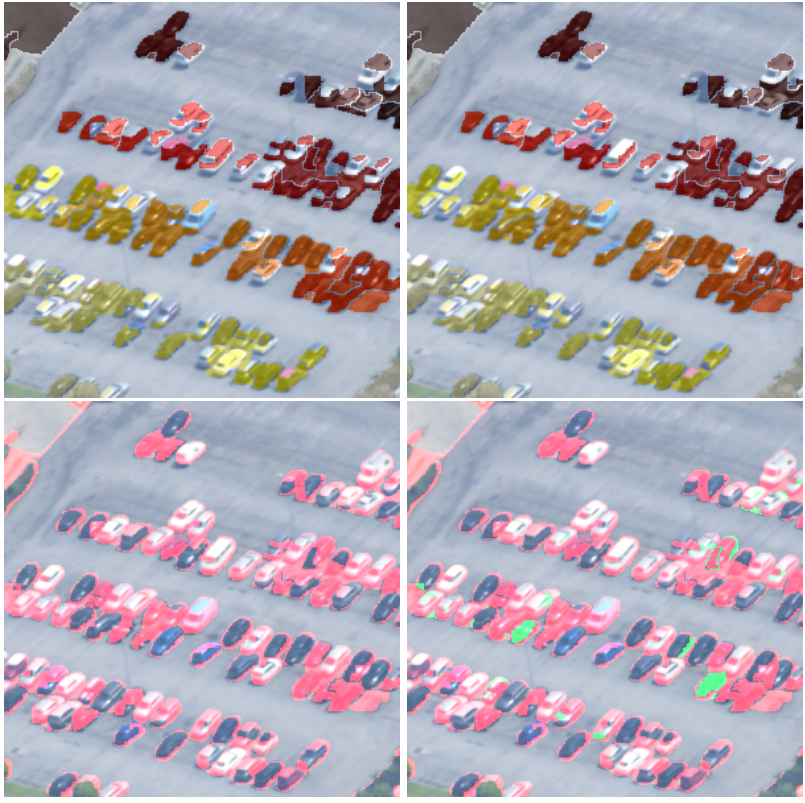

Figure 4. Top: Hitmap for the ucm alone thresholded at the highest segmentation covering (left) and hitmap with the presented algorithm (right). Bottom: The failed pixels marked in red. In the fused segmentation, the fails from the RGB segmentation not present in the fused one are marked in green

DSM is often over-smoothed. But it is also not affected by layover and foreshortening and thus enables a better distinction of these (predictable) areas.

The presented pipeline is based on the gPb-owt-ucm and extends the hierarchical segmentation to different sensors. But the oneway hierarchy also creates difficulties: Where RGB at the given threshold did not detect a boundary, SAR and DSM also could not. A system that enables weighted combination of all channels as a preparatory step, and not only RGB, might be better suited. $\mathrm{Pb}$ is already older, and newer segmentations for RGB look promising both in terms of speed and segmentation results (Isola et al., 2014). However, for our scene, other results, including the intended extensions of the $\mathrm{Pb}$ like the global $\mathrm{Pb}(\mathrm{gPb})$, produced worse results. The weighting used for the SAR and DSM is a very simple one. For SAR, more sophisticated models exist that were already used for segmentation with good results (Rodrigues et al., 2016).

Looking at the DSM in figure 2, it is not yet understood why the height information was not used during the segmentation, as the cars are clearly distinguishable: In the oblique view, a great amount of the information is spread out vertically, e.g. building façades. For the sake of generality, the height information was not used for this experiment.

Inaccuracies in the projection pose a serious problem. In this experiment, this was circumvented by a precise referencing of the DSM. But even then, in addition to the single channels displaying different features, small misregistrations render the segmentation inaccurate and the contours in the channels in different places.

\section{CONCLUSION}

We have presented a new fusion scheme for the segmentation of decimeter-resolution SAR and RGB data acquired from the same platform, based on the gPb-owt-ucm pipeline, which performs better than RGB-only input segmentation. The benefits are still small, but have the potential for further gains. The main challenge faced when fusing high-resolution SAR and optical data is the different geometric representations. These were partially overcome by first generating a DSM from the optical data, then focussing the SAR data onto it, and finally projecting it into the RGB camera reference frame. Using this method, the DSM also helped to guide the segmentation.

\section{REFERENCES}

Arbelaez, P., Maire, M., Fowlkes, C. and Malik, J., 2011. Contour detection and hierarchical image segmentation. IEEE Transactions on Pattern Analysis and Machine Intelligence 33(5), pp. 898-916.

Boulch, A., Le Saux, B. and Audebert, N., 2017. Unstructured point cloud semantic labeling using deep segmentation networks. In: Proceedings of the Eurographics 2017 Workshop on 3D Object Retrieval, Eurographics Association. to appear.

Frioud, M., Wahlen, A., Wellig, P. and Meier, E., 2014. Processing of MIRANDA35 FMCW-SAR Data using a Time-Domain Algorithm. In: Proc. European Conference on Synthetic Aperture Radar (EUSAR), VDE.

Gaetano, R., Moser, G., Poggi, G., Scarpa, G. and Serpico, S. B., 2008. Region-based classification of multisensor optical-sar images. In: Proc. IEEE International Geoscience and Remote Sensing Symposium (IGARSS), Vol. 4, IEEE, pp. IV-81.

Isola, P., Zoran, D., Krishnan, D. and Adelson, E. H., 2014. Crisp boundary detection using pointwise mutual information. In: Proc. European Conference on Computer Vision (ECCV), Springer, pp. $799-814$.

Rodrigues, F. Á., Neto, J. R., Marques, R. P., de Medeiros, F. S. and Nobre, J. S., 2016. Sar image segmentation using the roughness information. IEEE Geoscience and Remote Sensing Letters 13(2), pp. 132-136

Schack, L., Soergel, U. and Heipke, C., 2015. Persistent scatterer aided facade lattice extraction in single airborne optical oblique images. ISPRS Annals Photogrammetry, Remote Sensing and Spatial Information Sciences 2(3), pp. 197.

Sportouche, H., Tupin, F. and Denise, L., 2011. Extraction and three-dimensional reconstruction of isolated buildings in urban scenes from high-resolution optical and sar spaceborne images. IEEE Transactions on Geoscience and Remote Sensing 49(10), pp. 3932-3946.

Verdoliva, L., Gaetano, R., Ruello, G. and Poggi, G., 2015. Optical-driven nonlocal sar despeckling. IEEE Geoscience and Remote Sensing Letters 12(2), pp. 314-318.

Voisin, A., Krylov, V. A., Moser, G., Serpico, S. B. and Zerubia, J., 2014. Supervised classification of multisensor and multiresolution remote sensing images with a hierarchical copula-based approach. IEEE Transactions on Geoscience and remote sensing 52(6), pp. 3346-3358.

Wegner, J. D. and Soergel, U., 2008. Bridge height estimation from combined high-resolution optical and sar imagery. In: Proc. ISPRS Symposium, Beijing, IAPRS, Vol. 37, pp. 1071-1076. 